\title{
MANIPULACIÓN DE OBJETOS DESCONOCIDOS ANALIZANDO LOCALMENTE SU FORMA PARA OPTIMIZAR LAS FUERZAS DE PRENSIÓN
}

\author{
Andrés Montaño - Raúl Suárez \\ Institut d'Organitzaciò i Control de Sistemes Industrials (IOC) \\ Universitat Politècnica de Catalunya (UPC), Barcelona. \\ andres.felipe.montano@upc.edu - raul.suarez@upc.edu
}

\begin{abstract}
Resumen
Los enfoques propuestos para la manipulación de objetos se basan, generalmente, en el conocimiento de las características del objeto manipulado. En este trabajo se presenta un enfoque geométrico que permite manipular un objeto desconocido basándose únicamente en información cinemática y táctil. Los datos de los sensores táctiles y los valores articulares del robot, que se obtienen durante la manipulación, se usan para reconocer la forma del objeto (al menos la curvatura local de la superficie en contacto durante la manipulación), buscando optimizar las fuerzas de contacto. El enfoque propuesto ha sido implementado y probado usando la mano robótica Schunk Dexterous Hand (SDH2). Los resultados experimentales presentados muestran la validez del enfoque.
\end{abstract}

Palabras clave: Manipulación, prensión, sensores táctiles.

\section{INTRODUCCIÓN}

La manipulación de objetos es un problema de gran interés en robótica. Este problema ha sido abordado con diferentes enfoques, pero muchos de ellos no son aplicables en escenarios reales donde el entorno y el objeto manipulado son desconocidos. La inclusión de sensores táctiles en las manos robóticas [1] ayuda a obtener información del contacto con el objeto manipulado, incrementando las capacidades del robot. Además, la información táctil es útil en aplicaciones donde otras fuentes de información, como por ejemplo la visión artificial, no están disponibles. En general, la información táctil ayuda a reducir la incertidumbre durante la manipulación, permitiendo aplicaciones robóticas en el mundo real [12].

La manipulación tiene generalmente tres objetivos: la optimización de la configuración de la mano robótica, buscando una configuración deseada; la optimización de la prensión, buscando incrementar su robustez; y la optimización de la configuración del objeto manipulado buscando alcanzar una configuración deseada [6]. La optimización de las fuerzas de contacto está relacionada intrínsicamente con estos objetivos.

Muchos de los enfoques propuestos están basados en esquemas de control, pero estos no suelen considerar la realimentación táctil, por ejemplo, se ha propuesto un esquema de control de posición para cambiar la posición del objeto manipulado [16], pero la falta de realimentación sensorial limita su aplicabilidad. También se ha propuesto un esquema de control posición-fuerza para mover el objeto manipulado siguiendo una trayectoria dada [3], pero la evaluacíon del enfoque se llevó a cabo únicamente en simulación, introduciendo ruido en las medidas del sensor para emular un entorno real. La exploración y reconocimiento de la superficie del objeto manipulado han sido abordadas usando un controlador de posiciónfuerza para controlar el deslizamiento de los dedos [11]. Otro enfoque aborda el problema de rotación del objeto circulares sin conocer su radio usando un controlador de torque para los dedos [15].

En un trabajo previo de los autores [5], se propuso un enfoque para reconocer la forma del objeto usando información cinemática y de contacto obtenida durante la manipulación, esta información se usa para calcular invariantes de distancia que forman una firma del objeto manipulado. En otro trabajo [6], se propuso un esquema de manipulación que realiza una búsqueda de las configuraciones de la mano que mejoran diferentes índices de calidad relacionados con los tres objetivos más comunes de la manipuación. El enfoque propuesto en este trabajo pretende ofrecer un procedimiento geométrico sencillo capaz de combinar el reconocimiento de la curvatura local de la superficie del objeto con la estrategia de manipulación, permitiendo de este modo una optimización de las fuerzas de contacto durante la manipulación.

El problema abordado en este trabajo es la optimización de la fuerza de contacto cuando 
se manipula un objeto desconocido, entendiendo por tal que no hay conocimiento previo de su forma y que por tanto no se usa al iniciar la manipulación. La manipulación se realiza usando dos dedos de una mano robótica, cuyas yemas tienen sensores táctiles que indican la ubicación del contacto y la fuerza que aplican sobre el objeto manipulado. La presión realizada es similar a la de un humano cuando usa los dedos pulgar e índice [4]. Los movimientos de los dedos están limitados a un plano pero aun así se pueden realizar tareas como, por ejemplo, inspeccionar un objeto o hacer coincidir dos piezas para realizar un ensamblaje [17]. El cálculo de la configuración de la prensión inicial está fuera del alcance de este trabajo, pero puede realizarse usando diferentes planificadores de prensiones [13]. Aquí se considera desconocida la ubicación de los puntos de contacto sobre la superficie del objeto, su masa y centro de masa, el coeficiente de fricción, y las fuerzas externas aplicadas sobre el objeto [14]. Por lo tanto, el sistema únicamente tiene información de los valores articulares de los dedos, dada por los sensores internos de la mano, y de los puntos y fuerzas de contacto sobre los dedos, dada por los sensores táctiles. No hay otras fuentes externas de información, como por ejemplo un sistema de visión para reconocer la forma y localización del objeto. Se asume un coeficiente de fricción real mayor que un valor mínimo prefijado, ya que las yemas de los dedos están cubiertas de goma.

El objetivo del enfoque propuesto es determinar una secuencia de configuraciones de la mano que permitan una manipulación segura del objeto y, al mismo tiempo, obtener información de la forma del objeto para optimizar las fuerzas aplicadas durante la manipulación. Tanto los movimientos de manipulación como la identificación de la forma del objeto (al menos la curvatura local) se realizan iterativamente usando únicamente infomación cinemática y táctil, y esta misma información se utiliza para optimizar las fuerzas aplicadas sobre el objeto manipulado.

\section{MODELADO DE LA PRENSIÓN}

La Figura 1 muestra el modelo geométrico de una presión usando dos dedos. Cada dedo $f_{i}$, $i \in\{1,2\}$, es una cadena cinemática de $n_{i}$ links de longitud $l_{i j}, j \in\left\{1, . ., n_{i}\right\}$ siendo $n_{i}$ el número de grados de libertad $(g d l)$ de cada dedo. La posición relativa de cada link con respecto al link anterior se denota por el valor articular $q_{i j}$. Una configuración del dedo está dada por el conjunto de valores articulares $\boldsymbol{q}_{i}=\left\{q_{i 1}, \cdots, q_{i n_{i}}\right\}$. Una

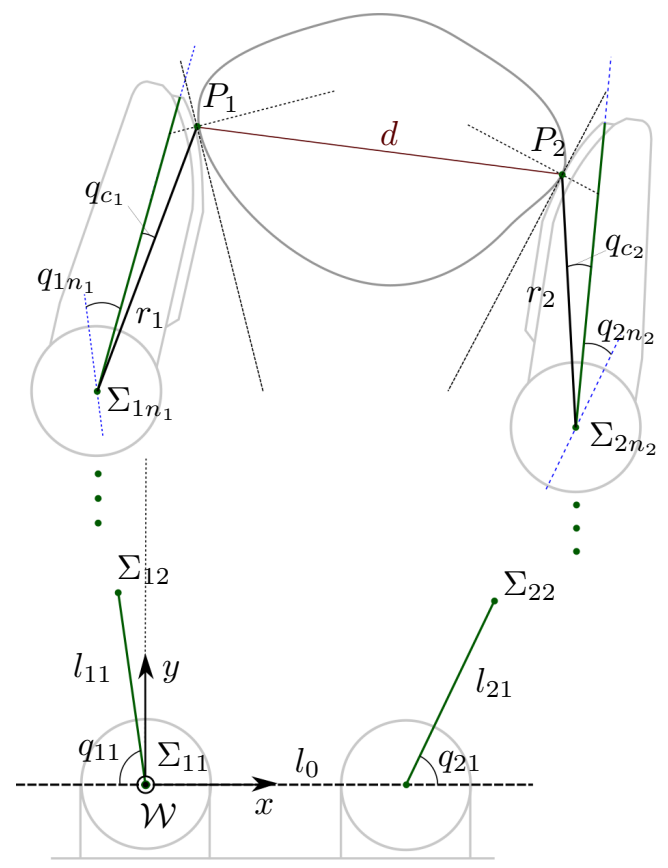

Figura 1: Modelo geométrico de una presión con dos dedos.

configuración de la mano es la concatenación de las configuraciones de los dos dedos $\boldsymbol{Q}=\left\{\boldsymbol{q}_{1}, \boldsymbol{q}_{2}\right\}$. La base de cada link tiene asociado un sistema de referencia $\Sigma_{i j}$.

El sistema de referencia absoluto $\mathcal{W}$ está ubicado en la base del dedo $f_{1} . \quad P_{i}$ es la posición del punto de contacto en el dedo $f_{i}$ con respecto a $\mathcal{W}$. Las medidas del sensor táctil están referenciadas al sistema de referencia del sensor $\mathcal{S}$. Para incluir la información de los sensores táctiles en la cinemática de la mano se usa un link virtual, que añade a cada dedo un $g d l$ extra, $q_{c_{i}}$, no controlable. La longitud $r_{i}$ del link virtual es la del segmento entre el origen del sistema de referencia $\Sigma_{i n_{i}}$ y el punto de contacto $P_{i}^{s}$, con el sudíndice $s$ indicando que $P_{i}$ está expresado en el sistema de referencia del sensor $\mathcal{S}$. Los puntos de contacto $P_{i}$ se calculan usando la cinemática directa con la información de los dedos y del link virtual. La distancia euclídea entre los puntos de contacto $P_{1}$ y $P_{2}$ es

$$
d\left(P_{1}, P_{2}\right)=\sqrt{\left(P_{1_{x}}-P_{2_{x}}\right)^{2}+\left(P_{1_{y}}-P_{2_{y}}\right)^{2}}
$$

\section{RECONOCIMIENTO DE LA CURVATURA DEL OBJETO}

La curvatura local del objeto se determina en línea aplicando una regresión circular sobre el conjunto de puntos de contacto que se producen durante la manipulación. Sea $\mathcal{O}$ un sistema de referencia asociado al objeto, cuyo origen 
y orientación están definidos por el punto de contacto $P_{1}^{w}$ y la orientación del sistema de referencia absoluto $\mathcal{W}$, ambos en la prensión inicial. Para determinar la curvatura del objeto, los puntos de contacto deben pasarse del sistema de referencia absoluto $\mathcal{W}$ al sistema de referencia del objeto $\mathcal{O}$. Para realizar este cambio de sistema de referencia se usa también información obtenida durante la manipulación: la distancia $d_{k}$ entre los puntos de contacto, la distancia recorrida sobre la yema del dedo $L_{i_{k}}$ (calculada a partir de medidas consecutivas de los puntos de contacto sobre el sensor táctil), y la orientación del objeto $\gamma_{k}$.

La orientación del objeto $\gamma_{k}$ se calcula mediante geometría básica usando la información obtenida de los sensores táctiles y la cinemática de los dedos. La orientación $\gamma_{k}$ resultante en la presión inicial se considera como $\gamma_{0}=0$, y se usa como referencia para la orientación del objeto. Cuando la punta del dedo tiene forma circular, la orientación del objeto $\gamma_{k}$ se puede aproximar mediante [9],

$$
\gamma_{k} \approx \frac{R}{d_{k}} \sum_{j=1}^{n_{1}}\left(q_{1 j_{\gamma_{0}}}-q_{1 \mathrm{j}_{k}}\right)-\sum_{j=1}^{n_{2}}\left(q_{2 \mathrm{j}_{\gamma_{0}}}-q_{2 j_{k}}\right)
$$

Dadas $L_{i_{k}}$, los puntos de contacto $P_{i_{k}}^{o}$ se encuentran sobre sendas circunferencias con radios $L_{1_{k}}$ y $L_{2_{k}}$, y centradas en los puntos de contacto $P_{1_{0}}^{o}$ y $P_{2_{0}}^{o}$, respectivamente; es decir los primeros puntos de contacto en la prensión inicial como se muestra en la Figura 2. Estas circunferencias pueden describirse como (el subíndice $k$ se omite en las siguientes expresiones para mejorar la legibilidad):

$$
\begin{gathered}
\left(P_{1_{x}}^{o}\right)^{2}+\left(P_{1_{y}}^{o}\right)^{2}=L_{1}^{2} \\
\left(P_{2_{x}}^{o}-d \cos \gamma\right)^{2}+\left(P_{2_{y}}^{o}-d \sin \gamma\right)^{2}=L_{2}^{2}
\end{gathered}
$$

Además, los puntos $P_{1}^{o}$ y $P_{2}^{o}$ deben satisfacer

$$
\begin{gathered}
d=\sqrt{\left(P_{2_{x}}^{o}-P_{1_{x}}^{o}\right)^{2}+\left(P_{2_{y}}^{o}-P_{1_{y}}^{o}\right)^{2}} \\
\left(P_{2_{y}}^{o}-P_{1_{y}}^{o}\right)=\left(P_{2_{x}}^{o}-P_{1_{x}}^{o}\right) \tan \gamma
\end{gathered}
$$

Las Ecuaciones (3), (4), (5) y (6) se resuelven para $P_{1_{x}}^{o}, P_{2_{x}}^{o}, P_{1_{y}}^{o}$ y $P_{2_{y}}^{o}$ resultando:

$$
\begin{gathered}
P_{1_{x}}^{o}=P_{2_{x}}^{o}-\rho \\
P_{1_{y}}^{o}=P_{2_{y}}^{o}-\beta \\
P_{2_{x}}^{o}=\frac{-b \pm \sqrt{b^{2}-4 a c}}{2 a} \\
P_{2_{y}}^{o}=\sqrt{L_{2}^{2}-\left(P_{2_{x}}^{o}-d \cos \gamma\right)^{2}}+d \sin \gamma
\end{gathered}
$$

donde

$$
\rho=\sqrt{\frac{d^{2}}{1+(\tan \gamma)^{2}}} \quad \beta=\tan \gamma \sqrt{\frac{d^{2}}{1+(\tan \gamma)^{2}}}
$$

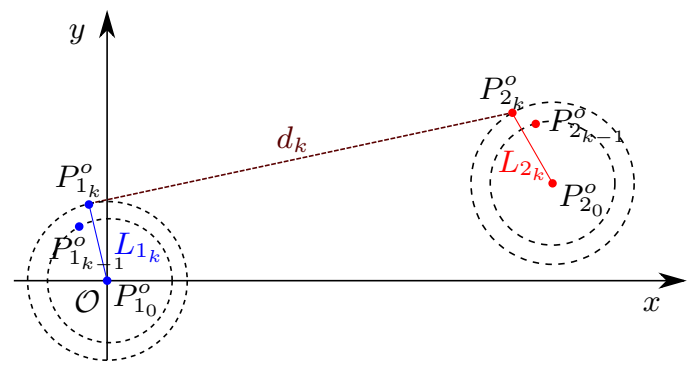

Figura 2: Regiones circulares de radio $L_{1_{k}}$ y $L_{2_{k}}$, donde se encuentran los puntos que pertenecen a la superficie del objeto.

$$
\begin{aligned}
a & =(-2 d \sin \gamma+2 \beta)^{2}+(-2 d \cos \gamma+2 \rho)^{2} \\
b & =-2 d \cos \gamma(-2 d \sin \gamma+2 \beta)^{2} \\
& -2 \psi(-2 d \cos \gamma+2 \rho) \\
& +2 d \sin \gamma(-2 d \sin \gamma+2 \beta)(-2 d \cos \gamma+2 \rho) \\
c & =(d \cos \gamma)^{2}(-2 d \sin \gamma+2 \beta)^{2} \\
& +\psi^{2}-2 \psi d \sin \gamma(-2 d \sin \gamma+2 \beta) \\
& +(d \sin \gamma)^{2}(-2 d \sin \gamma+2 \beta)^{2} \\
& -L_{2}^{2}(-2 d \sin \gamma+2 \beta)^{2}
\end{aligned}
$$

$\psi=L_{2}^{2}-L_{1}^{2}-(d \cos \gamma)^{2}-(d \sin \gamma)^{2}+\rho^{2}+\beta^{2}$

Una descripción detallada del procedimiento para identificar la forma del objeto se puede encontrar en [5], aunque en este trabajo la información de la curvatura del objeto se usa solo para calcular las configuraciones objetivo de la mano en lugar de para identificar por completo el modelo del objeto.

Sea $S_{P_{i}^{o}}$ el conjunto de $m$ puntos de contacto $P_{i}^{o}$ obtenidos durante la manipulación. El objetivo de la regresión circular es determinar el círculo $C_{i}^{o}$ con centro en el punto $\left(c_{i_{x}}^{o}, c_{i_{y}}^{o}\right)$ y radio $r_{c_{i}^{o}}$ que minimize la suma del cuadrado de las distancias desde cada punto en $S_{P_{i}^{o}}$ a $C_{i}^{o}$, es decir, los puntos deben encontrarse lo más cercanos posible a $C_{i}^{o}$ [2]. La asunción de formas circulares se basa en el hecho de que "la mayoría de los objetos en el mundo están formados por arcos circulares y segmentos rectos" [10]. Nótese que un segmento recto puede verse como un arco circular con un radio suficientemente grande. Los círculos $C_{i}^{o}$ se pasan al sistema de referencia $\mathcal{W}$ llevando el punto central $\left(c_{i_{x}}^{o}, c_{i_{y}}^{o}\right)$ a un punto separado por una distancia $r_{c_{i}^{o}}$ en la dirección normal al punto de contacto $P_{i_{k}}$, como se muestra en la Figura 4. Entonces $C_{i}^{w}$ se usa para calcular unos puntos de contacto virtuales $P_{i_{k+1}}^{*}$ usados en el procedimiento de manipulación descrito en la Sección 4. 


\section{MANIPULACIÓN USANDO INFORMACIÓN TÁCTIL}

El proceso de manipulación empieza con el objeto ya sujeto en la mano y tiene como entradas la fuerza de contacto deseada $F_{d}$ y la orientación del objeto deseada $\gamma_{d}$. Este es un procedimiento iterativo que calcula las configuraciones de la mano que permiten rotar el objeto buscando alcanzar la orientación deseada y al mismo tiempo controla la fuerza de prensión. En cada iteración $k$ se ejecutan los siguientes pasos:

1) Cálculo del estado actual de la prensión. Este paso incluye el cálculo de la posición de los puntos de contacto $P_{1_{k}}$ y $P_{2_{k}}$, que se obtienen a partir de los valores articulares $\boldsymbol{q}_{i}$ y de la información del link virtual aplicando la cinemática directa de los dedos. También se calcula la fuerza de prensión $F_{k}$ como el promedio de las fuerzas de contacto $F_{1_{k}}$ y $F_{2_{k}}$ medidas en cada una de las yemas de los dedos. El promedio de ambas fuerzas de contacto minimiza posibles errores en las medidas de las fuerzas. Se cálcula además la orientación del objeto $\gamma_{k}$. Recuérdese que el objetivo de la manipulación es reducir el error de orientación $\varepsilon_{o}$, calculado como la diferencia entre la orientación deseada $\gamma_{d}$ y la orientación actual $\gamma_{k}$ del objeto, es decir, $\varepsilon_{o}=\gamma_{d}-\gamma_{k}$.

2) Cálculo de los puntos virtuales de contacto. Asumiendo que para calcular correctamente la curvatura local del objeto son necesarios al menos $m$ puntos de su superficie, durante las primeras $m$ iteraciones no se tiene información de la forma del objeto y los dos puntos de contacto virtuales $P_{1_{k+1}}^{*}$ y $P_{2_{k+1}}^{*}$ se calculan considerándolos como el desplazamiento del punto de contacto actual en la superficie del sensor sobre un camino circular virtual de diámetro $d_{k}$, centrado en el punto medio $R_{k}$ entre los puntos $P_{1_{k}}$ y $P_{2_{k}}$, es decir los puntos de contacto virtuales están dados por (ver Figura 3):

$$
\begin{aligned}
& P_{1_{k+1_{x}}}^{*}=R_{k_{x}}-\left(d_{k} / 2\right) \cos \left(\gamma_{k+1}\right) \\
& P_{1_{k+1_{y}}}^{*}=R_{k_{y}}-\left(d_{k} / 2\right) \sin \left(\gamma_{k+1}\right) \\
& P_{2_{k+1}}^{*}=R_{k_{x}}+\left(d_{k} / 2\right) \cos \left(\gamma_{k+1}\right) \\
& P_{2_{k+1_{y}}}^{*}=R_{k_{y}}+\left(d_{k} / 2\right) \sin \left(\gamma_{k+1}\right)
\end{aligned}
$$

con

$$
\gamma_{k+1}=\gamma_{k}+\tanh \left(\varepsilon_{o}\right) \Delta \gamma
$$

donde $\Delta \gamma>0$ se escoje empíricamente y suficientemente pequeña para asegurar movimientos pequeños del objeto en cada iteración, y la función tanh se usa para limitar la ganancia para valores grandes de $\gamma_{k}$.

Después de $m$ iteraciones (es decir, $k>m$ ), $P_{1_{k+1}}^{*}$ y $P_{2_{k+1}}^{*}$ se calculan considerándolos como

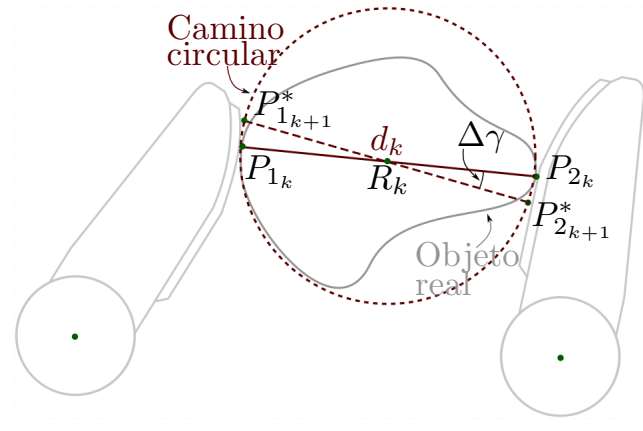

Figura 3: Movimientos usados para la optimización de la orientación del objeto. $P_{1_{k+1}}^{*} \mathrm{y}$ $P_{2_{k+1}}^{*}$ son calculados sobre un camino circular virtual con diámetro $d_{k}$ y centrado en $R_{k}$.

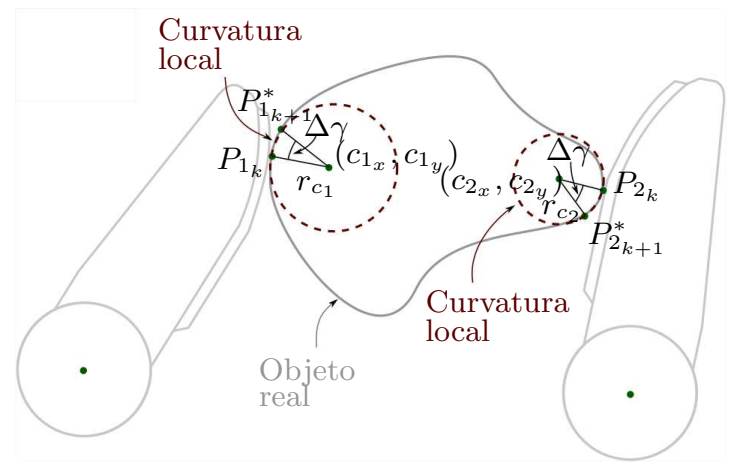

Figura 4: Movimientos usados para la optimización de la orientación del objeto mejorando la fuerza de contacto. $P_{1_{k+1}}^{*}$ y $P_{2_{k+1}}^{*}$ son calculados sobre caminos circulares generados mediante regresiones circulares usando los puntos de contacto previos.

el resultado del desplazamiento de los puntos de contacto sobre el sensor a lo largo de caminos circulares definidos por círculos $C_{i}$ centrados en $\left(c_{i_{x}}, c_{i_{y}}\right)$ y de radio $r_{c_{i}}$, estos círculos se obtienen usando una regresión circular como se describió en la Sección 3, es decir, el camino circular virtual inicial es reemplazado por los caminos descritos por los círculos calculados usando la información obtenida durante la manipulación (ver Figura 4). Por lo tanto, $P_{1_{k+1}}^{*}$ y $P_{2_{k+1}}^{*}$ ahora están dados por,

$$
\begin{aligned}
& P_{1_{k+1}}^{*}=c_{1_{x_{k}}}-\left(r_{c_{1_{k}}}\right) \cos \left(\gamma_{k+1}\right) \\
& P_{1_{k+1} y}^{*}=c_{1_{y_{k}}}-\left(r_{c_{1_{k}}}\right) \sin \left(\gamma_{k+1}\right) \\
& P_{2_{k+1_{x}}}^{*}=c_{2_{x_{k}}}+\left(r_{c_{2_{k}}}\right) \cos \left(\gamma_{k+1}\right) \\
& P_{2_{k+1} y}^{*}=c_{2_{y_{k}}}+\left(r_{c_{2_{k}}}\right) \sin \left(\gamma_{k+1}\right)
\end{aligned}
$$

3) Ajuste de la distancia entre puntos de contacto. Los movimientos de los dedos cambian la fuerza de contacto $F_{k}$. Si $F_{k}$ se incrementa puede producir daños en el objeto o en la mano, y si $F_{k}$ decrece el objeto se puede caer. Para reducir el error en la fuerza aplicada sobre el objeto $\varepsilon_{f}=F_{k}-F_{d}$, la 


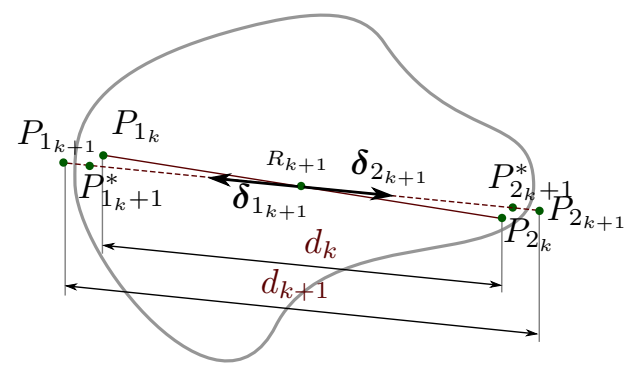

Figura 5: Ejemplo de cálculo de $P_{i_{k+1}}$ usando $P_{i_{k+1}}^{*}$ ajustando la distancia $d_{k}$ a $d_{k+1}$ cuando la fuerza de contacto $F_{k}$ es mayor que $F_{d}$.

distancia $d_{k}$ se ajusta en cada iteración haciendo $d_{k+1}=d_{k}+\Delta d$, con

$$
\Delta d= \begin{cases}2 \lambda\left(\varepsilon_{f}+\varepsilon_{f}^{2}\right) & \text { if } \varepsilon_{f} \leq 0 \\ \lambda \varepsilon_{f} & \text { if } \varepsilon_{f}>0\end{cases}
$$

donde $\lambda>0$ es una constante predefinida. La corrección del error negativo tiene mayor ganancia porque una potencial caída del objeto $\left(F_{k} \rightarrow 0\right)$ es más crítica que la aplicación de una fuerza de contacto grande $\left(F_{k} \gg F_{d}\right)$.

4) Cálculo de los puntos de contacto objetivo. Los puntos de contacto virtuales $P_{1_{k+1}}^{*}$ y $P_{2_{k+1}}^{*}$ se ajustan para obtener los puntos de contacto objetivo $P_{1_{k+1}}$ y $P_{2_{k+1}}$ separados por una distancia $d_{k+1}$,

$$
P_{i_{k+1}}=R_{k+1}+\frac{d_{k+1}}{2} \boldsymbol{\delta}_{i_{k+1}}, \quad i \in\{1,2\}
$$

donde $R_{k+1}$ es el punto central entre $P_{1_{k+1}}^{*}$ y $P_{2_{k+1}}^{*}$, y $\boldsymbol{\delta}_{i_{k+1}}$ es el vector unitario que va desde $R_{k+1}$ a $P_{i_{k+1}}^{*}$ (ver Figura 5). La configuración de la mano $\boldsymbol{Q}_{k+1}=\left\{\boldsymbol{q}_{1_{k+1}}, \boldsymbol{q}_{2_{k+1}}\right\}$ se obtiene de los puntos de contacto objetivo $P_{1_{k+1}}$ y $P_{2_{k+1}}$ usando la cinemática inversa de los dedos.

5) Verificación de las condiciones de finalización. El proceso de manipulación termina cuando se activa cualquiera de las siguientes condiciones de finalización: a) El error de orientación es cero, es decir, el objeto se encuentra en la orientación deseada; b) La orientación actual del objeto no mejora después de un número predeterminado de iteraciones; c) La prensión esperada con los puntos de contacto calculados no satisface las restricciones de fricción; y d) Los puntos de contacto calculados no pertenecen al espacio de trabajo de los dedos.

6) Movimiento de los dedos. Finalmente, si no se ha activado ninguna condición de finalización, la mano se mueve a $\boldsymbol{Q}_{k+1}$ para que los dedos alcancen los puntos de contacto objetivo $P_{1_{k+1}}$ y $P_{2_{k+1}}$ y roten el objeto. Después del movimiento de los dedos empieza una nueva iteración.

\section{EXPERIMENTACIÓN}

La mano robótica usada en la experimentación es la Schunk Dexterous Hand (SDH2). Esta mano tiene siete $g d l$, dos en cada uno de sus tres dedos y uno adicional que permite la rotación de dos de los dedos para que trabajen de forma opuesta uno del otro. Una descripción detallada de la cinemática de la mano se puede encontrar en [7]. La mano tiene un sistema de sensores táctiles en las yemas de los dedos y en las falanges proximales. En este trabajo solo se usan las yemas de los dedos para la manipulación. Los sensores tienen 68 texels, y cada uno mide una fuerza de prensión de hasta 3 N. Los sensores táctiles proveen información sobre el baricentro de la región de contacto y la sumatoria de las fuerzas medidas sobre todos los texels en contacto. Entonces, para usar el modelo tradicional de contacto puntual [8], el baricentro de la región de contacto se usa como punto de contacto, y la sumatoria de fuerzas se considera como la fuerza aplicada en el dicho punto [18].

El procedimiento propuesto ha sido probado satisfactoriamente con diferentes tipos de objetos. A continuación se describe un ejemplo ilustrativo utilizando un objeto con dos curvaturas constantes y diferentes en las regiones de contacto. La fuerza de prensión deseada se fijó en $F_{d}=4 \mathrm{~N}$. Se asumió un coeficiente de fricción $\mu=0.4$ menor que el valor físico real considerando que las yemas de los dedos están cubiertas de goma. La constante para ajustar la distancia entre los puntos de contacto de acuerdo con la Ecuación (20) se fijó en $\lambda=0.25 \mathrm{~mm}$, la constante para cambiar la orientación de objeto en $\Delta \gamma=0.25$ grados, $\mathrm{y}$ el número de puntos para calcular la curvatura local de objeto en $m=30$. Las consignas de orientación $\gamma_{d}$ dadas al sistema fueron -5, 5, -10 y 10 grados, en ese orden. Para ilustrar el efecto de usar las curvaturas locales en el cálculo de las configuraciones objetivos de la mano, primero se llevó a cabo la manipulación del objeto sin usar las curvaturas locales y luego se repitió el proceso teniéndolas en cuenta según se van calculando durante la manipulación. De esta forma pueden compararse las fuerzas de contacto realizando la misma manipulación con y sin consideración de las curvaturas locales. La fuerza promedio registrada cuando se manipuló el objeto sin considerar las curvaturas locales fue de $4.04 \mathrm{~N}$ con una varianza de $0.3476 \mathrm{~N}$, mientras que la registrada cuando se consideraron las curvaturas locales fue de $3.86 \mathrm{~N}$ con una varianza de $0.1471 \mathrm{~N}$.

La Figura 6 muestra los resultados de la manipulación. Las Figuras 6a y $6 \mathrm{~b}$ muestran imágenes del objeto en la prensión inicial y en la configuración final cuando el objeto ha 


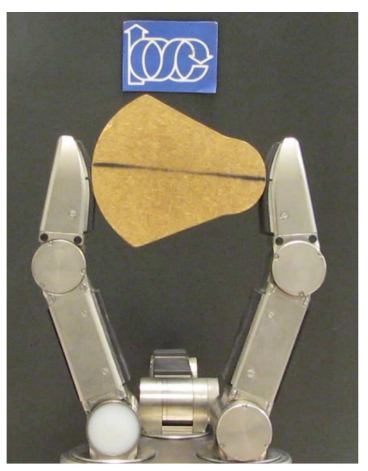

a)

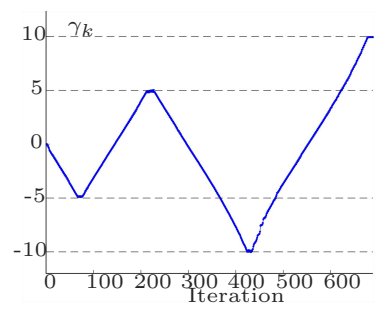

c)

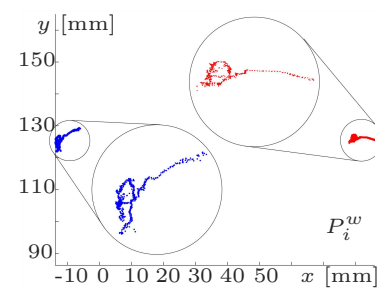

e)

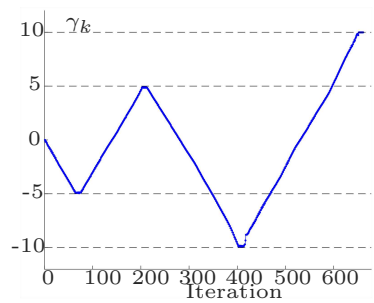

g)

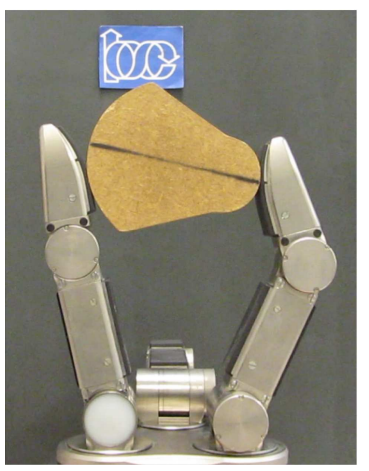

b)

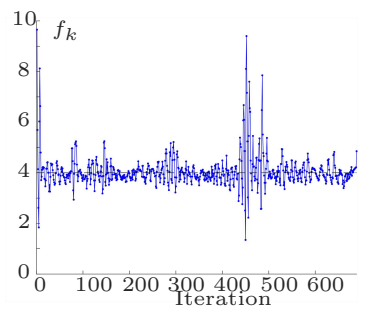

d)

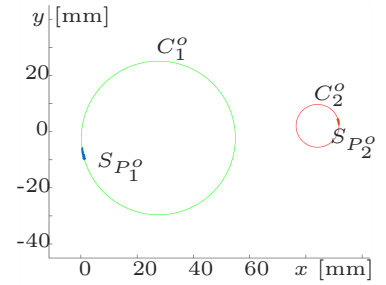

f)

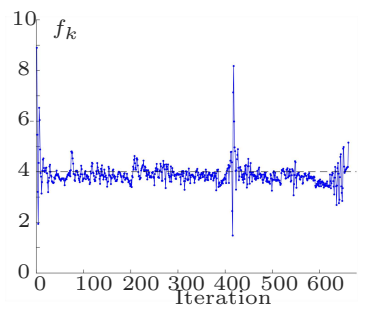

h)
Figura 6: Ejemplo: a) Prensión inicial; b) Configuración de la mano cuando el objecto ha rotado 10 grados; c) Evolución de la rotación $\gamma_{k}$ del objeto (en grados) sin usar identificacón de curvaturas; d) Evolución de la fuerza de contacto $F_{k}$ (en Newtons) sin usar identificacón de curvaturas; e) Puntos de contacto resultantes $P_{i}^{w}$ con ampliación de las regiones de interés; f) Puntos de contacto $P_{i}^{o}$ y últimos círculos $C_{i}^{o}$ calculados mediante la regresión circular, con ampliación de las regiones de interés; g) Evolución de la rotación $\gamma_{k}$ del objeto (en grados) usando identificacón de curvaturas; h) Evolución de la fuerza de contacto $F_{k}$ (en Newtons) usando identificacón de curvaturas. sido rotado 10 grados. Las Figuras $6 \mathrm{c}$ y $6 \mathrm{~d}$ muestran la evolución de la orientación del objeto $\gamma_{k}$ y de la fuerza de contacto $F_{k}$, durante la manipulación del objeto sin utilizar el cálculo de las curvaturas locales. La Figura 6e muestra los puntos de contacto $P_{i}^{w}$ utilizados para calcular las curvaturas locales mientras se manipulaba por segunda vez el objeto. La Figura 6f muestra los puntos de contacto $P_{i}^{o}$ y los últimos círculos $C_{i}^{o}$ calculados. Las Figuras $6 \mathrm{~g}$ y $6 \mathrm{~h}$ muestran la evolución de la orientación del objeto $\gamma_{k}$ y de la fuerza de contacto $F_{k}$, cuando se manipuló el objeto utilizando las curvaturas locales para calcular las configuraciones objetivo de la mano.

\section{CONCLUSIONES Y TRABAJO FUTURO}

En este trabajo se ha propuesto un procedimiento para optimizar las fuerzas de contacto durante la manipulación de objetos desconocidos usando información táctil y cinemática generada durante la manipulación. El enfoque propuesto identifica las curvaturas locales del objeto manipulado y calcula en línea las configuraciones de la mano que permiten cambiar la orientación del objeto de forma segura. Los requisitos para su implementación en un sistema robótico son el conocimiento de la cinemática de la mano, un control de posición de los dedos y la disponibilidad de información táctil.

Una extensión natural del enfoque propuesto es la optimización de las fuerzas de contacto cuando la manipulación del objeto persigue otros objetivos, es decir, la optimización de la configuración de la mano o de la calidad de la prensión. También se considera la extensión a manipulaciones usando más de dos dedos.

\section{Agradecimientos}

Este trabajo ha sido parcialmente financiado por el Gobierno de España a través del proyecto DPI2016-80077-R.

\section{English summary}

Manipulation of Unknown Objects with a Local Analysis of its Shape to Optimize the Manipulation Forces

\footnotetext{
Abstract

Object manipulation approaches usually require the knowledge of the manipulated object. In this paper it is presented a simple geometric approach to manipulate unknown objects, the approach requires only kinematic and tactile in-
} 
formation obtained during manipulation. Tactile and kinematic data are used to determine the object shape. Tha hand configurations computed using the object shape optimize the grasping force. The proposed approach has been implemented and tested using the Schunk Dexterous Hand (SDH2). Experimental results are presented to illustrate the efficiency of the approach.

keywords: Manipulation, grasping, tactile sensors.

\section{Referencias}

[1] A. Bicchi. Hands for Dextrous Manipulation and Powerful Grasping: a Difficult Road Towards Simplicity. IEEE Transactions on Robotics and Automation, 16(6):652-662, 2000.

[2] N. Chernov. Circular and Linear Regression: Fitting Circles and Lines by Least Squares, volume 117 of Monographs on Statistics and Applied Probability. Chapman \& Hall / CRC, 2010.

[3] Q. Li, R. Haschke, H. Ritter, and B. Bolder. Towards unknown objects manipulation. In IFAC Proceedings Volumes, pages 289-294, 2012.

[4] C. L. MacKenzie and T. Iberall. The Grasping Hand, volume 104. Elsevier, 1994.

[5] A. Montaño and R. Suárez. Object shape reconstruction based on the object manipulation. In Proceedings of IEEE International Conference on Advanced Robotics, pages 1-6, 2013.

[6] A. Montaño and R. Suárez. Unknown object manipulation based on tactile information. In Proceedings of IEEE/RSJ International Conference on Intelligent Robots and Systems, pages 5642-5647, 2015.

[7] A. Montaño and R. Suárez. Commanding the object orientation using dexterous manipulation. In Advances in Intelligent Systems and Computing, volume 418, pages 6979. Springer Verlag, 2016.

[8] V.-D. Nguyen. Constructing Force- Closure Grasps. The International Journal of Robotics Research, 7(3):3-16, jun 1988. ISSN 0278-3649.

[9] R. Ozawa, S. Arimoto, P. Nguyen, M. Yoshida, and J. H. Bae. Manipulation of a circular object in a horizontal plane by two finger robots. 2004 IEEE International Conference on Robotics and Biomimetics, pages 517-522, 2004.
[10] W. A. Perkins. A model-based vision system for industrial parts. IEEE Transactions on Computers, C-27(2):126-143, Feb 1978. ISSN 0018-9340. doi: 10.1109/TC.1978.1675046.

[11] R. Platt. Learning and Generalizing ControlBased Grasping and Manipulation Skills. $\mathrm{PhD}$ thesis, University of Massachusetts Amherst, 2006.

[12] M. Prats, A. P. D. Pobil, and P. J. Sanz. Robot Physical Interaction through the combination of Vision, Tactile and Force Feedback - Applications to Assistive Robotics, volume 84 of Springer Tracts in Advanced Robotics. Springer, 2013.

[13] C. Rosales, R. Suárez, M. Gabiccini, and A. Bicchi. On the synthesis of feasible and prehensile robotic grasps. In Proceedings of IEEE International Conference on Robotics and Automation, pages 550-556, may 2012.

[14] W. Shaw-Cortez, D. Oetomo, C. Manzie, and P. Choong. Tactile-based blind grasping: A discrete-time object manipulation controller for robotic hands. IEEE Robotics and Automation Letters, 3(2):1064-1071, April 2018. doi: 10.1109/LRA.2018.2794612.

[15] S. K. Song, J. B. Park, and Y. H. Choi. Dual-fingered stable grasping control for an optimal force angle. IEEE Transactions on Robotics, 28(1):256-262, 2012.

[16] K. Tahara, S. Arimoto, and M. Yoshida. Dynamic object manipulation using a virtual frame by a triple soft-fingered robotic hand. In Proceedings of IEEE International Conference on Robotics and Automation, pages 4322-4327, 2010.

[17] Y. P. Toh, S. Huang, J. Lin, M. Bajzek, G. Zeglin, and N. S. Pollard. Dexterous telemanipulation with a multi-touch interface. In Proceedings of IEEE-RAS International Conference on Humanoid Robots, pages 270-277, 2012.

[18] H. Wörn and T. Haase. Force approximation and tactile sensor prediction for reactive grasping. In Proceedings of World Automation Congress, pages 1-6, 2012.

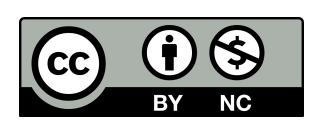

(c) 2018 by the authors. Submitted for possible open access publication under the terms and conditions of the Creative Commons Attribution CC-BY-NC 3.0 license (http://creativecommons.org/licenses/by-nc/3.0/). 